\title{
Design eines praxisorientierten und wissensbasierten Cyber-Physischen Systems für industrielle Rüstvorgänge
}

\author{
Nils Darwin Abele' • Sven Hoffmann • Aparecido Fabiano Pinatti de Carvalho • Marcus Schweitzer · \\ Volker Wulf · Karsten Kluth
}

Angenommen: 12. November 2021 / Online publiziert: 21. Dezember 2021

@ Der/die Autor(en) 2021

\section{Zusammenfassung}

Industrielle Rüstvorgänge sind durch ihren zentralen und zeitkritischen Charakter innerhalb eines Produktionsprozesses elementar. Optimierungsbestrebungen dieser Prozesse erfolgen insbesondere hinsichtlich des Zeitaufwandes und der Ressourcenschonung. In diesem Zusammenhang findet die Unterstützung des Menschen durch digitale Technologien in Form von Cyber-Physischen Systemen (CPS) weniger Beachtung. Der vorliegende Beitrag greift diese Thematik auf und trägt zur Weiterentwicklung von CPS bei. Auf Grundlage einer umfassenden ethnographischen Studie wurden Praktiken von Maschineneinrichtern im Zuge von Rüstvorgängen an Biegemaschinen identifiziert. Die Erkenntnisse dieser Studie münden in ein übergreifendes Wissenstransfermodell, das die Ableitung von Gestaltungs- bzw. Designimplikationen für CPS im Rüstkontext erleichtert und optimiert. Auf Grundlage eines Vergleichs von technischen Möglichkeiten mit den im Modell erfassten praxisrelevanten Anforderungen an den Wissens- und Erfahrungstransfer konnte ein neuer auf Augmented Reality (AR) und Sensortechnologie beruhender methodischer Ansatz zur Erfassung und Weitergabe von propositionalem und prozessualem Wissen, eingebettet in verkörperten Handlungen, realisiert werden.

Praktische Relevanz: Die Forschungsarbeiten bringen den Stand der Technik bei der Gestaltung digitaler Technologien bzw. CPS zur Unterstützung von Menschen voran, die mit Umrüstprozessen industrieller Produktionsmaschinen betraut sind. Es konnten für den Austausch von Fachwissen insbesondere hinsichtlich der Kontextualität und Kommunikation unter Berücksichtigung kollaborativer Arbeit und sozialer Rahmenbedingungen Fortschritte erzielt werden, die gleichzeitig neue Forschungsrichtungen zu diesem Thema eröffnen.

Schlüsselwörter Augmented Reality · Cyber-Physische Systeme · Design Case Studies · Industrielle Rüstprozesse · Wissenstransfer

\section{Design of a practical and knowledge-based cyber-physical system for industrial set-up processes}

\begin{abstract}
Due to their central and time-critical character, industrial set-up processes are elementary within a production process. Efforts to optimize these processes are made in particular with regard to the time required and the conservation of resources. Less considered in this context is the support of humans by digital technologies in the form of cyber-physical systems (CPS). This paper addresses this issue and contributes to the further development of CPS. Based on a comprehensive ethnographic study practices of machine setters were identified in the course of set-up operations on bending machines. The findings of this study result in an overarching knowledge transfer model that facilitates and optimizes the derivation of design implications for CPS in the set-up context. Based on a comparison of technical possibilities with the practice-relevant requirements for knowledge and experience transfer captured in the model, a new methodological approach based on augmented reality (AR) and sensor technology for capturing and transferring propositional and process knowledge embedded in embodied actions could be realized.
\end{abstract}

Nils Darwin Abele

darwin.abele@uni-siegen.de
1 Fachgebiet Arbeitswissenschaft/Ergonomie, Universität Siegen, Paul-Bonatz-Straße 9-11, 57076 Siegen, Deutschland 
Practical Relevance: The research advances the state of the art in the design of digital technologies or CPS to support people involved in set-up processes of industrial production machines. In particular, progress has been made with respect to contextuality and communication for sharing expertise, taking into account collaborative work and social frameworks, while opening up new research directions on this topic.

Keywords Augmented Reality · Cyber-Physical Systems · Design Case Studies · Industrial Set-up Processes · Knowledge Transfer

\section{Einleitung}

Industrielle Rüstvorgänge umfassen vor dem Start eines Produktionszyklus eine Reihe von vorbereitenden Maßnahmen an (Fertigungs-)Maschinen oder Werkzeugen. Dieser Prozess beeinflusst den Produktionsfluss durch seinen zentralen und zeitkritischen Charakter unmittelbar. Seine Optimierung ist der Schlüssel zur Maximierung einer effizienten Fertigung und zum bestmöglichen Handling kundenspezifischer Produkte bzw. kleiner Auftrags- und Liefergrößen (De Carvalho et al. 2018).

Durch eine zunehmende Globalisierung, spezifische Kundenwünsche, intensiven Wettbewerb sowie kurze Innovations- und Produktlebenszyklen resultiert daraus ein Flexibilitätserfordernis, welche sowohl für Klein- und mittelständische Unternehmen (KMU) als auch für große Unternehmen gilt und direkte Auswirkungen auf industrielle Rüstvorgänge hat (Hoffmann et al. 2019; Ludwig et al. 2016). Einerseits besteht die Notwendigkeit, den Ressourceneinsatz zu reduzieren. Andererseits werden immer vielfältigere Qualitätsanforderungen gestellt. Dieser Flexibilitätsbedarf bezieht sich auch auf die Mitarbeiterebene, indem flexible Kompetenzen zur Bewältigung heterogener Rüstvorgänge gefordert werden. Die daraus resultierende Komplexität geht mit einer wissensintensiven Umgebung einher (Ackerman et al. 2013; Argote et al. 2000).

Mehrere Forschungsbereiche beschreiben die Schwierigkeit, Wissen und Fähigkeiten aus der realen Welt zu erfassen, zu beschreiben und letztlich zu demonstrieren, sodass auf dieses Wissen zurückgegriffen werden kann (Schmidt 2012; Nonaka und Takeuchi 1995). Die Unterscheidung von „Knowing how" und „Knowing that" liegt in ihrer Propositionalität (Ryle 1945, 2009). Während sich Letzteres verbalisieren bzw. propositional ausdrücken lässt, erfordert ersteres hingegen kein theoretisches Verständnis (Ackerman et al. 2013; Schmidt 2012). Polanyi (1967) erweitert diese Diskussion um die Begrifflichkeiten „,implizites“ oder ,stillschweigendes"Wissen. Es soll deshalb u. a. gezeigt werden, dass tazites Wissen nicht zwangsläufig stillschweigend ist, sondern vielmehr in Gesprächen artikuliert wird oder sich in Gesten, Blicken oder Aktionen manifestiert. Das geschieht jedoch unter höchst kontingenten und lokalen Umständen (Randall et al. 1996). Qualitative Ansätze verhelfen in diesem Zusammenhang zur „Sichtbarmachung“ von „Know how" und „unsichtbaren“ Arbeitspraktiken (Clarke et al. 2003).

Nichtsdestotrotz ist es keineswegs trivial, das in Praktiken eingebettete, nicht-propositionale Wissen (selbst) zu erfassen. Neben zeitlichen Engpässen (Schmidt 2012) können die Privatsphäre und die durch Wissen erworbenen Alleinstellungsmerkmale (Nonaka 1994) der Wissensarchivierung entgegenstehen. Weiterhin sind didaktische Barrieren hinsichtlich einer generischen Darstellung der Informationen zu überwinden, um „Know how“ für jeden Einzelnen verständlich zu machen (Schmidt 2012).

Digitale Technologien treiben in Form von Informations- und Kommunikationstechnologien (IKT) in sämtlichen Branchen Veränderungen und Innovationen an und durchdringen zunehmend den Alltag westlicher Gesellschaften. Eine kundenorientierte sowie auf digitale Wertschöpfung ausgerichtete Unternehmensphilosophie zielt auf smarte digitale, datenbasierte Produkte bzw. Dienstleistungen ab. Eine Lösung, die an diese Zielsetzung anknüpft, ist das „Internet of Things" (IoT). Das IoT vernetzt physische Objekte mit einer virtuellen Umgebung. Diese Verbindung wird durch das Zusammenspiel von Sensorik und dem Internet bzw. einer Cloud-Plattform zugunsten eines allgegenwärtigen und echtzeitnahen Datenzugriffs ermöglicht, z.B. durch Ansätze wie „Big Data“, „Cognitive Computing“, „Pervasive Computing“ und „Ubiquitous Computing“. „Industrie 4.0" ist eine Bewegung, die aus der Forderung der deutschen Bundesregierung nach einer ,vierten industriellen Revolution" entstanden ist - inspiriert durch den Einsatz von neuen sogenannten Cyber-Physischen Systemen (CPS), die in Fertigungskontexten auch als Cyber-Physische Produktionssysteme (CPPS) bekannt sind (Paelke und Röcker 2015). Das „Industrial Internet of Things“ (IIoT) wird, insbesondere im englischsprachigen Bereich, oft als Synonym für Industrie 4.0 verwendet. Beide Begriffe stehen im beschriebenen Kontext für die Implementierung des IoT in die industrielle Produktionsumgebung. Zusammen mit dem Aufkommen von CPS können Analysen, Wissensextraktionen und -archivierungen sowie Diagnosen ökonomischer Prozesse auf Grundlage großer und vernetzter Datenmengen durchgeführt und damit die Verflechtung wirtschaftlicher Infrastrukturen noch stärker vorangetrieben werden (Zidek et al. 2020). 
Neue Technologien, wie auf Augmented Reality (AR) basierende CPS in Kombination mit vielfältigen Möglichkeiten des I(I)oT, können dazu verhelfen, die beschriebene Problematik zu adressieren (De Carvalho et al. 2018). AR ist als neue Visualisierungsart, die ortsbezogene Informationen schnell und einfach übertragen kann, potenziell dazu geeignet, Wissen und Expertise zu erfassen und zu teilen (Ackerman et al. 2013).

Trotz des in der Literatur identifizierten technischen Potentials moderner Technologien wurden nur wenige Erkenntnisse hinsichtlich der Bedürfnisse und Anforderungen der Nutzer publiziert. Die Arbeiten des Forschungsprojekts „Cyberrüsten 4.0“ (Abele et al. 2016) griffen diese Thematik auf, um ein prototypisches aber erfolgsversprechendes AR- und sensorbasiertes Unterstützungssystem für den Wissens- und Erfahrungsaustausch im Rahmen industrieller Rüst- bzw. Einrichtprozesse zu konzipieren. In Kombination mit prozessrelevanten und kodifizierten Informationen über eine Maschine sowie über Werkzeug- und Materialeigenschaften kann die Unterstützungssystematik zusammen mit geeigneten sensorischen Eingangswerten und Simulationen helfen, die Rüstvorgänge effizienter zu gestalten. Dazu wurde im Rahmen einer ethnographischen Studie eine Analyse der Praktiken von Maschineneinrichtern im Zuge von Rüstvorgängen von Umform- bzw. Biegemaschinen durchgeführt. Dabei sollte eruiert werden, wie Augmented Reality und sensorbasierte CyberPhysische Systeme wichtige methodische Veränderungen in der Erfassung und Aufbereitung von Wissen bewirken können (De Carvalho et al. 2018). Weiterhin wurde ein Wissenstransfermodell entwickelt, das sowohl die Abstraktion übergeordneter Forschungselemente als auch die potenziell damit verbundenen Designaktivitäten umfasst (Hoffmann et al. 2019). Dieses dient im Sinne einer transdisziplinären Kommunikation einer Kanalisierung der beschriebenen Thematik innerhalb der Forschungsbereiche der ComputerSupported Cooperative Work (CSCW), der Human-Computer Interaction (HCI) sowie der Arbeitswissenschaft bzw. Ergonomie.

\section{Wissens- und Erfahrungstransfer im industriellen Kontext}

Wissensmanagement ist eine wichtige strategische Ressource, durch die Wettbewerbsvorteile am Markt generiert werden können (Ackerman et al. 2013; Argote et al. 2000). Viele Studien aus dem Bereich des CSCW untersuchten die Rolle von Informationen in organisatorischen und sozialen Kontexten mit einem Fokus auf die Weitergabe bzw. gemeinsame Nutzung von Wissen durch sachkundige Mitarbeiter in sicherheitskritischen Arbeitsumgebungen und im administrativen Bereich (Ackerman et al. 2013; Randall et al. 1996). Der Fachbereich der HCI betrachtet den Themenbereich insbesondere im Hinblick auf die Gestaltung digitaler Visualisierungstechnologien für den Wissenstransfer. Zugehörige Studien bezogen sich insbesondere auf das Lehren und Lernen (Patterson et al. 2014; Tergan und Keller 2005) oder Informationsüberlastungen (,Information Overload“", Mengis und Eppler 2012).

Keine dieser Studien untersucht jedoch die Rolle der gemeinsamen Nutzung von wissensintensiven Praktiken, Fachwissen und des Wissenstransfers in industriellen Rüstprozessen. Vielmehr liegt der Schwerpunkt oftmals auf der Ausarbeitung von Modellen, die sich auf eine organisatorische Wissensschöpfung oder auf Motivationsaspekte zur Realisierung eines erfolgreichen Wissenstransfers beziehen (Nonaka et al. 2000). Die primäre Problematik hinsichtlich einer gemeinsamen Nutzung von Wissen besteht darin, das implizite Wissen zu explizieren (De Carvalho et al. 2018; Ackerman et al. 2013; Schmidt 2012). Ackerman und Halverson (2004) weisen in diesem Zusammenhang auf die Bedeutsamkeit der Aneignung eines detaillierten empirisch fundierten Verständnisses der mit dem Wissenstransfer verbundenen Praktiken hin. Praktiken werden als „Wege des Handelns" betrachtet und setzen zumeist kontextabhängiges Wissen voraus (Schmidt 2014). Darüber hinaus spielt jedoch auch nicht-propositionales Wissen, bspw. in Form verkörperter bzw. nicht-verbalisierbarer Handlungen (,embodied actions“), eine bedeutende Rolle (Gallagher 2010).

\section{3 „Embodied Action“}

Forschungsarbeiten bzw. Begriffsdefinitionen zu „Embodied Action" reichen von Merleau-Pontys Philosophie der Verkörperung (Gallagher 2010) über die „Philosophischen Untersuchungen“ (Wittgenstein 1953) bis hin zu ethnomethodologischen Sichtweisen (Lynch 1997). Wissen und Handeln, das sich zwangsläufig in ,verkörperten“ Aktionen ausdrückt, sind miteinander verbunden. Ein wesentlicher Forschungsaspekt ist jedoch darauf ausgerichtet, wie sich Wissen im Handeln widerspiegelt und wie verkörpertes Handeln in der Praxis in möglichst hoher Qualität dargestellt werden kann.

Neben einem wechselseitigen Einfluss von Aktion und Interaktion in der virtuellen und physischen Welt (Heath und Luff 1991) können sich verkörperte Handlungen auch im Umgang mit physischen Artefakten manifestieren, z.B. bei der Erstellung physischer Repräsentationen, wie das Schreiben und Zeichnen auf Papier, oder der tatsächliche Gebrauch physischer Objekte, wie das Arbeiten mit Computern, Lesen etc. (Brown und Duguid 1994). In Bezug auf andere Subjekte sind das Senden und Empfangen von ,Zeichen“ in Form visueller Indikatoren wesentlich. Dazu ge- 
hören u.a. spezifische Körperhaltungen, Gestik und Mimik sowie orale bzw. akustische Indikatoren, d.h. Sprechen und andere (externe) Geräusche (Kendon 1990). Die Unterstützung derartiger „Embodied Actions“ ist weitreichend und bedarf neben der (einfachen) Erfassung propositionaler Inhalte (z. B. über Videoaufnahmen) auch neuartiger technologischer Hilfsmittel, beispielsweise in Form von Sensorik.

\section{Augmented Reality und Cyber-Physische Systeme}

AR-Technologien können das in verkörperten Handlungen eingebettete Wissen aufzuzeichnen und $\mathrm{zu}$ visualisieren (Zidek et al. 2020; Pollalis et al. 2017). Grundsätzlich werden die hardwareseitigen AR-Ausgabetechnologien in Head-Mounted Displays (HMDs, Datenbrillen), Handheld Displays (Smartphones und Tablets) und Spatial Displays (Projektoren) unterschieden. Unterschiedliche Trackingverfahren (markerbasiertes und markerloses Tracking) verhelfen $\mathrm{zu}$ einer möglichst genauen Darstellung der perspektivischen Überlagerung von realen und digitalen Inhalten in Echtzeit (Dörner et al. 2019). In wissensintensiven Umgebungen ist der schnelle, uneingeschränkte und kontextspezifische Zugang zu Informationen ein vielversprechendes Mittel zur Unterstützung von Lernprozessen (Klopfer et al. 2005). Erkenntnisse aus der Literatur deuten auch darauf hin, dass AR-Tools die Fähigkeiten und die Wahrnehmung der Nutzer durch eine potenziell nachhaltige Optimierung der Arbeitsvorgänge verbessern können. Sowohl forschungsinduzierte als auch industrielle Beispiele sind in Form von AR-basierten Anleitungen bzw. Instruktionen, Fehlerdiagnosen oder jobspezifischen Trainings im Rahmen von Qualitätssicherungsmaßnahmen (FragaLamas et al. 2018; Frigo et al. 2016), Montageprozessen (Hegenberg und Schmidt 2021; Loch et al. 2016; Hořejší 2015; Ong et al. 2008), Wartungsarbeiten (Lee und Akin 2011) oder Schweißarbeiten (Park et al. 2007) zu finden.

Trotz der Arbeit von Tang et al. (2007), welche die Rolle der Verkörperung in Bezug auf Gruppeneffekte unter Berücksichtigung gemischter Präsenz untersuchten, bestehen in der Literatur keine Anhaltspunkte für eine wissensbasierte Nutzung von AR-Systemen in Bezug auf erfasste verkörperte Handlungen.

AR- und Sensortechnologien können, unter Berücksichtigung eines Einsatzes im sozio-technischen Kontext, potenziell zahlreiche verschiedene Prozesse unterstützen und erleichtern. CPS sind in diesem Zusammenhang Ansätze zum innovativen Handling wissensintensiver Prozesse zugunsten effizienter Arbeitspraktiken (Paelke und Röcker 2015). Sie stellen Systeme aus eng gekoppelten physischen und virtuellen bzw. digitalen Komponenten dar, die Software, Hardware, Sensoren und Aktoren integrieren (Lee et al.
2015). Ihre charakteristischen Merkmale sind die Vernetzung verschiedener Produktionskomponenten, wie Maschinen oder Werkzeuge, sowie die sie charakterisierenden Datensätze (Gallagher 2010). Die Interaktion mit solchen Systemen erfolgt über eine Mensch-Maschine-Schnittstelle, die durch konventionelle PC-Schnittstellen, Touchscreens oder AR-basierte Technologien implementiert werden kann. Eine Untersuchung, inwieweit solche Systeme den Austausch von Wissen und Expertise unterstützen können, indem sie das in verkörperten Handlungen eingebettete Wissen sammeln, ist bis dato nicht bekannt.

Lee et al. (2015) stellen für CPS eine Struktur und Architektur vor, die von der Datenerfassung über die Analyse bis hin zur endgültigen Wertschöpfung reicht und als Leitfaden für die Umsetzung solcher Systeme in der Industrie dienen soll. Praktische Anwendungen und Techniken ergänzen die theoretische Architektur mit praxisnahen Umsetzungsmöglichkeiten. Mit dem Design und der Implementierung von CPS gehen aber auch Herausforderungen einher, die u.a. durch eine erhöhte technische Komplexität die Notwendigkeit neuer Interaktionskonzepte sowie dem Mangel an Prototyping- und Testwerkzeugen charakterisiert sind (Paelke und Röcker 2015; Monostori 2014).

\section{Modellbasiertes Design für den Wissenstransfer}

Die Literatur zu Modellentwicklungen ist vielfältig. Sie reicht von einfachen Anwendungen (Benford et al. 1993) über die Entwicklung von Kommunikationsmodellen (DeMichelis und Grasso 1994) bis hin zur Untersuchung des Spielraums für die Entwicklung generischer Modelle zur Unterstützung von Kollaboration und Koordination (Mainwaring und Lee 2017). Nicht zuletzt wird auch die Unterstützung des Wissenstransfers bei kooperativer Arbeit thematisiert (Divitini et al. 1993).

Modellbasiertes Design fußt insbesondere in den o.g. Themengebieten auf ethnographischen Erkenntnissen. Entsprechende Ansätze setzen dabei Prozesse (Holtzblatt und Jones 2017), formale Artefakte oder Modelle (Salvador und Mateas 1997) oder grafische Darstellungen (Diggins und Tolmie 2003) in den Mittelpunkt. Eine Reihe von Ansätzen zielen auch auf die Modellierung von Wissenstransferprozessen ab, wie beispielsweise das sogenannte Spiral- oder SECI-Modell (Socialization, Externalization, Combination, Internalization) von Nonaka und Takeuchi (1995) zur Modellierung der Beschaffung und Weitergabe von Wissen oder das Modell des „Knowledge Sharing“ von Peinl (2006), das ebenfalls den intraorganisationalen Wissensaustausch thematisiert. Raelin (1997) erweitert diese Sichtweise auf das Lernen, indem er in Anlehnung an Nonaka und Takeuchi (1995) zwischen individuellem und kol- 
lektivem Lernen unterscheidet. Beide Sichtweisen werden wiederum in explizite und implizite Anteile untergliedert, sodass sich der Lernprozess letztlich in theoretische und praktische Aspekte aufteilt.

Ein weiteres in diesen Zusammenhang einzuordnendes (lernbezogenes) Modell ist die „Cognitive Load Theory“ (CLT, Sweller 1994). Die Theorie befasst sich im Zuge der Modellierung und Gestaltung von Wissenssystemen im Wesentlichen mit dem Lernen im Rahmen komplexer Aufgaben. Die CLT geht davon aus, dass Lernende bei der Bearbeitung neuer Probleme nur über ein begrenztes Arbeitsgedächtnis verfügen, d.h. ein Kurzzeitgedächtnis zur Aufnahme von Informationen, die für die Ausführung einer aktuellen Aufgabe notwendig sind. Die Generierung neuer Schemata sowie die Automatisierung bestehender Schemata ermöglicht dem Akteur die Freisetzung seines Arbeitsgedächtnis. Das Arbeitsgedächtnis weist drei verschiedene Arten der Belastung auf. Die Menge der benötigten Informationen und die Interaktion mit ihnen wird als intrinsische Belastung (1) bezeichnet. Die extrinsische Last (2) wird durch Informationen auferlegt, welche weder die Ableitung neuer Schemata begünstigen noch die Automatisierung erleichtern. Die „Germane load“ (3) bezieht sich auf die Erstellung neuer oder automatisierter Schemata. Ein Schlüsselaspekt dieses Ansatzes ist, dass verschiedene Belastungsarten unterschiedliche Auswirkungen auf die Generierung eines Lernprozesses und die Weitergabe von Wissen haben. In diesem Beitrag wird dieser Sachverhalt in Anlehnung an De Carvalho et al. (2018) insbesondere mit der Fragestellung in Verbindung gebracht, wie CPS die Erfassung und Visualisierung von nicht-propositionalem Wissen bestmöglich unterstützen können.

\section{Methodische Vorgehensweise zur Konzipierung eines Cyber-Physischen Systems zur Rüstprozessunterstützung}

Um zu verstehen, wie CPS die Bedürfnisse der Nutzer im Rahmen industrieller und komplexer Rüstvorgänge als adäquate Hilfestellung unterstützen können, wurde eine Designfallstudie (Wulf et al. 2015) durchgeführt. Die Designfallstudie stellt dazu einen nutzerzentrierten Rahmen für die Gestaltung und Entwicklung sozio-technischer Systeme dar. Innerhalb von drei verschiedenen Phasen erfolgt zunächst - basierend auf einer empirischen Analyse des Handlungsfeldes mitsamt des Nutzerkontexts, der Praktiken und den daraus resultierenden Bedürfnissen (1) - ein Designprozess (2), innerhalb dessen neue Lösungen auf Grundlage der identifizierten Bedürfnisse entworfen werden. Die Designfallstudie wird mit einer Aneignungs- bzw. Evaluierungsphase (3) abgeschlossen, in der die Lösungen in einem realen Szenario getestet und im Hinblick auf de- ren Auswirkungen sowie hinsichtlich aller resultierenden sozialen Veränderungen bewertet werden.

\subsection{Empirische Analyse des Handlungsfeldes}

Zunächst wurde eine empirische Analyse des anwendungsbezogenen Handlungsfelds, d.h. des Rüstprozesses, durchgeführt. Sie deckt als solche diejenigen Nutzerpraktiken auf, die das Potenzial aufweisen, durch technische Lösungen unterstützt zu werden. Die Beschreibung der Praktiken, die technologische, organisatorische und soziale Perspektiven aufweisen, erfolgt minutiös. Die Analyse bedient sich dabei qualitativer empirischer Methoden, um eine Reflektion der potenziellen Nutzer mit Bezug auf ihre Praxissituation anzustreben. Daraus resultieren Anforderungen in Form von Gestaltungsimplikationen für einen möglichen technischen Lösungsansatz.

Im Forschungsprojekt „Cyberrüsten 4.0“ wurde ein ethnographischer Ansatz gewählt, um die Praktiken und den sozialen Kontext von Maschineneinrichtern und Maschineneinrichterinnen sowie deren mögliche Auswirkungen auf die Gestaltung neuer Technologien zu verstehen. Ethnographische Forschung zielt in erster Linie darauf ab, „das Unsichtbare sichtbar zu machen“ (Goodwin 1994), indem die implizite, soziale und kulturelle Organisation beschrieben wird, welche wiederum die Aktivitäten der Teilnehmer charakterisiert (Anderson-Levitt 2006). Im Zuge der Analyse wurde eine interaktionsanalytische Sichtweise eingenommen, welche die sequentielle Organisation von Aktivitäten und die Denkprozesse derjenigen betont, die sie ausführen, sowie derer, die sie zu verstehen versuchen. In dieser Sichtweise führen die Akteure, d.h. die Einrichter:innen, durch ihre kollaborative Arbeit oft Aktivitäten aus, die eng miteinander verbunden sind, ohne dass sie ihre Handlungen explizit besprechen (Heath und Luff 1991). Vereinfacht ausgedrückt wird gegenseitiges Verständnis erreicht, ohne auf explizite Anweisungen zurückzugreifen.

Für eine sehr engmaschige Analyse eines komplexen Rüstvorgangs wurden, ergänzend zu „Shadowing“-Sitzungen, d.h. zu Beobachtungen der Probanden für eine bestimmte Zeit, ohne diese zu beeinflussen, zur Erhebung von In-situ-Daten zu Arbeitspraktiken und sozialer Interaktion detailgenaue Videoaufnahmen benötigt, unter Zuhilfenahme einer Eye-Tracking-Technologie (Tobii Pro Lab). EyeTracking verhilft durch die Erfassung des Blickes bzw. der Fixationen dazu, durch Heatmap-Analysen ein intensives Verständnis zu den durchgeführten Handlungen sowie zu den im Rahmen der Rüstvorgänge genutzten Werkzeugen und Montagehilfsmitteln aufzubauen. Zusätzlich dazu wurden die Teilnehmer aufgefordert, während der Aufzeichnung dem ,Think Aloud“-Prinzip zu folgen, um sie mithilfe von „lautem Denken“ dahingehend zu provozieren, ihre derzeit ausgeführte Tätigkeit zu verbalisieren und damit In- 
Tab. 1 Teilnehmer der empirischen Studie

Table 1 Participants of the empirical study

\begin{tabular}{|c|c|c|c|c|c|c|c|c|}
\hline \multirow{2}{*}{$\begin{array}{l}\text { Teil- } \\
\text { nehmer }\end{array}$} & \multirow{2}{*}{$\begin{array}{l}\text { Inter- } \\
\text { view }\end{array}$} & \multicolumn{2}{|l|}{ Beobachtung } & \multirow{2}{*}{$\begin{array}{l}\text { Design- } \\
\text { Workshops }\end{array}$} & \multirow{2}{*}{$\begin{array}{l}\text { Vorl. Usability- } \\
\text { Tests }\end{array}$} & \multirow[t]{2}{*}{ Firma } & \multirow{2}{*}{$\begin{array}{l}\text { Fachliche Qualifizierung } \\
\text { (fachspezifisch: fs; fachfremd: ff) }\end{array}$} & \multirow{2}{*}{$\begin{array}{l}\text { Betriebszu- } \\
\text { gehörigkeit }\end{array}$} \\
\hline & & Eye-Tracking & Shadowing & & & & & \\
\hline T1 & $X$ & - & - & $X$ & - & A & Akad. Grad (ff) & $>10$ Jahre \\
\hline $\mathbf{T 2}$ & $X$ & - & - & - & - & A & Meisterschule (fs) & $>10$ Jahre \\
\hline T3 & $X$ & - & - & - & $\mathrm{X}$ & A & Meisterschule (fs) & $>10$ Jahre \\
\hline T4 & $\mathrm{X}$ & - & - & $X$ & - & A & Akad. Grad (ff) & $>10$ Jahre \\
\hline T5 & $\mathrm{X}$ & $X$ & - & - & - & A & Meisterschule (fs) & $<10$ Jahre \\
\hline T6 & $\mathrm{X}$ & $X$ & - & $\mathrm{X}$ & - & A & Meisterschule (fs) & $<10$ Jahre \\
\hline T7 & $X$ & $X$ & $\mathrm{X}$ & $X$ & $X$ & A & Ausbildung (fs) & $>10$ Jahre \\
\hline T8 & $\mathrm{X}$ & $X$ & $\mathrm{X}$ & $\mathrm{X}$ & - & $\mathrm{B}$ & Ausbildung (fs) & $<10$ Jahre \\
\hline T9 & $X$ & $X$ & - & $X$ & - & B & Ausbildung (fs) & $<10$ Jahre \\
\hline T10 & $X$ & $X$ & - & $X$ & - & $\mathrm{B}$ & Ausbildung (fs) & $<10$ Jahre \\
\hline T11 & $\mathrm{X}$ & $X$ & $\mathrm{X}$ & $X$ & - & $\mathrm{B}$ & Ausbildung (fs) & $<10$ Jahre \\
\hline T12 & $\mathrm{X}$ & - & - & $\mathrm{X}$ & - & $\mathrm{B}$ & Ausbildung (fs) & $<10$ Jahre \\
\hline T13 & $\mathrm{X}$ & - & - & $\mathrm{X}$ & - & $\mathrm{B}$ & Meisterschule (fs) & $>10$ Jahre \\
\hline T14 & $X$ & - & - & - & - & B & Akad. Grad (fs) & $>10$ Jahre \\
\hline T15 & $\mathrm{X}$ & - & - & - & - & B & Meisterschule (fs) & $>10$ Jahre \\
\hline T16 & $\mathrm{X}$ & - & - & - & - & $\mathrm{B}$ & Akad. Grad (fs) & $>10$ Jahre \\
\hline T17 & $\mathrm{X}$ & - & - & - & - & B & Akad. Grad (fs) & $>10$ Jahre \\
\hline T18 & $\mathrm{X}$ & - & - & - & - & $\mathrm{B}$ & Akad. Grad (fs) & $>10$ Jahre \\
\hline T19 & $\mathrm{X}$ & - & - & - & - & $\mathrm{B}$ & Akad. Grad (fs) & $<10$ Jahre \\
\hline T20 & $X$ & $X$ & $X$ & $X$ & $X$ & $\mathrm{C}$ & Ausbildung (fs) & $>10$ Jahre \\
\hline $\mathbf{T 2 1}$ & - & - & - & $X$ & $\mathrm{X}$ & $\mathrm{C}$ & Ausbildung (fs) & $<10$ Jahre \\
\hline $\mathbf{T} 22$ & $X$ & $X$ & - & - & - & $\mathrm{D}$ & Ausbildung (fs) & $<10$ Jahre \\
\hline $\mathbf{T 2 3}$ & $\mathrm{X}$ & $X$ & - & - & - & $\mathrm{D}$ & Meisterschule (fs) & $>10$ Jahre \\
\hline $\mathbf{T 2 4}$ & $X$ & $X$ & - & - & - & $\mathrm{E}$ & Ausbildung (fs) & $<10$ Jahre \\
\hline $\mathbf{T 2 5}$ & $\mathrm{X}$ & - & - & - & - & $\mathrm{E}$ & Akad. Grad (fs) & $>10$ Jahre \\
\hline
\end{tabular}

formationen über ihre Überlegungen im Verlauf des Rüstprozesses zu sammeln. Zusammen mit den transkribierten Kommentaren der Teilnehmer konnten so auch komplexe Prozessschritte in ihrer Gesamtheit erfasst und ausgewertet werden.

An der empirischen Studie nahmen insgesamt $25 \mathrm{Ar}-$ beitspersonen aus fünf verschiedenen KMUs (hier: Unternehmen A-D) im Alter zwischen 20 und 60 Jahren teil (vgl. Tab. 1). Alle Unternehmen waren mittelständisch und stellten über Biege- bzw. Tiefziehprozesse Kaltumformteile her. Die Teilnehmer hatten differente Positionen im Unternehmen inne (Konstruktionsingenieure, Maschineneinrichter, Meister bzw. Vorarbeiter, Produktdesigner, Produktionsingenieure, Prozessmanager, Qualitätsingenieure, Technische Vertriebsmitarbeiter), kamen aus unterschiedlichen Bildungsschichten (fachspezifischer oder fachfremde Qualifizierung in Form eines akademischen Grads, einer Ausbildung oder einer Meisterschule) und wiesen verschiedene Berufslaufzeiten (mehr oder weniger als 10 Jahre) auf. Somit konnte eine differenzierte Untersuchung zur Gestaltung der erforderlichen Technologie für ein breites Spektrum von Endnutzern realisiert werden. Um die Praktiken des Wissenstransfers granular untersuchen zu können, wurden Tiefen- bzw. Experteninterviews durchgeführt (Hermanowicz 2002). Diese wurden ebenfalls aufgezeichnet und transkribiert. Während die Beobachtungsphasen eine Dauer zwischen 45 und 90 min aufwiesen und die Shadowing-Sitzung zwischen 3 und $4 \mathrm{~h}$ andauerten, wurden die Interviews in 45 bis 120 min durchgeführt. Die Interviewtranskription erfolgte nach der Methode des „Intelligent Verbatim“, bei der sämtliche sprachliche Äußerungen Wort für Wort transkribiert werden, mit Ausnahme von nuschelnden bzw. unverständlichen Aussprachen (z.B. ,äh“ oder „,mh“), Füllwörtern (z.B. ,also“, ,ja“ oder „halt") und Versprechern (Hickley 2016).

Interviewtranskripte, Feldnotizen, Eye-Tracking-Aufnahmen und prozessrelevante firmeninterne Dokumente wurden einer thematischen Analyse unterzogen. Die Erkenntnisse aus den verschiedenen Datenartefakten wurden außerdem trianguliert, um ihre Vertrauenswürdigkeit als Qualitätskriterium für qualitative Forschung zu gewährleisten.

Aus der Analyse haben sich vier wesentliche Hauptthemen herausgestellt: der Workflow-Charakter eines industri- 
ellen Rüstprozesse (1), das gemischte Verhältnis von dynamischen und statischen Prozesselementen mit mechanischen und nicht-mechanischen Arbeitsvorgängen (2), der sehr wissensintensive Charakter des Prozesses (3) sowie die potenziellen Herausforderungen bei der Interaktion mit jeglicher digitaler Technologie während einer entsprechenden Tätigkeit (4). Das darauf basierende Analyseergebnis dieser rund zehnmonatigen Vorstudie resultiert schlussendlich in der Ableitung von Designimplikationen für AR-basierte CPS zur Unterstützung von Maschinenbedienern, die in Bezug auf kontextspezifische industrielle Rüstvorgänge Erfahrungs- bzw. Fachwissen festhalten und aufbereiten.

\subsection{Entwicklung einer innovativen Darstellungsform}

Die sich anschließende Phase umfasst neben der Anwendung nutzerzentrierter und partizipativer Designmethoden (Muller und Kuhn 1993) die Beschreibung spezifischer Designprozesse und der involvierten Akteure. Somit wird dem Aufbau bzw. der Gestaltung eines technisches Systemkonzepts Rechnung getragen. Auf Grundlage der empirischen Ergebnisse können folglich innovative IKT-Artefakte entwickelt werden, die sowohl produkt- als auch prozessspezifisch beschreibbar sind.

Die Designimplikationen wurden erarbeitet, indem die empirischen Ergebnisse mit der bestehenden Literatur zum Design von CPS in Verbindung gesetzt wurden. Die Designimplikationen haben demnach die Designaktivitäten der zweiten Phase kanalisiert. Hier wurde ein szenariobasiertes Design verwendet, um die partizipative Gestaltung zu unterstützen. Die Erstellung der Szenarien erfolgte auf Basis der Analyse der in der Vorstudie gesammelten Daten, die den Teilnehmern in einer Reihe von Design-Workshops wiederum als Diskussionsgrundlage diente. Diese Workshops wurden entsprechend mit repräsentativen Anwendern durchgeführt und folgten somit den Prämissen eines partizipativen Designansatzes, der die Einbeziehung der Teilnehmer in die Designentscheidungen hinsichtlich Funktionalität und Konzeption über die gesamte Designphase hinweg beinhaltet.

Basierend auf den identifizierten Anforderungen wurden zwei Szenarien ausgearbeitet, die mit Hilfe von Storyboards illustriert wurden. Deren Verwendung ist durch die Förderung von Diskussionen innerhalb der Design-Workshops und der Abstimmung mit den Teilnehmern begründet, wie bei der Gestaltung und Entwicklung der Systemunterstützung vorgegangen werden sollte.

Insgesamt wurden drei Design-Workshops durchgeführt. Die Design-Workshops dauerten zwischen vier bis acht Stunden an und wiesen eine Gruppengröße von jeweils sechs Teilnehmern auf. Die Design-Workshops wurden aufgezeichnet, transkribiert und ebenfalls einer thematischen Analyse unterzogen.

Nach den Design-Workshops erfolgte das „Rapid Prototyping" der jeweiligen Interfaces. Frühe Prototypen und die Technologie, die für das entworfene CPS ausgewählt wurde, durchliefen eine heuristische Evaluation (Nielsen und Molich 1990). Ziel war es dabei, bekannte Usability-Probleme zu beseitigen. Nach Verbesserungen, die sich aus der heuristischen Evaluation ergaben, wurden die Prototypen einer Reihe von Usability-Tests unterzogen. Einige Teilnehmer der Feldarbeit nahmen an diesen „Vorabtests“ teil (vgl. Tab. 1), damit sie dadurch weiterhin in den Designprozess eingebunden waren.

\subsection{Evaluierung in der Praxis}

Den Abschluss des methodischen Konstrukts bildet eine Längsschnittstudie zur Untersuchung und detaillierten Dokumentation des Einsatzes der entwickelten technologischen Artefakte innerhalb der sozialen Nutzersysteme. Das Design wird durch den Nutzer reflektiert und hinsichtlich der Aneignung des Artefakts bzw. der Wirkung auf den Nutzer innerhalb der Praxis überprüft. Dadurch kann das Ausmaß, inwieweit der entwickelte Lösungsansatz den Anforderungen der Nutzer gerecht wird, evaluiert und potenzielle Designänderungen erörtert werden.

Um die gewonnenen qualitativen Erkenntnisse verifizieren bzw. falsifizieren und schließlich erweitern zu können, wurden zusätzlich Teilstudien durchgeführt, die auch quantitative Komponenten einbeziehen (Abele und Kluth 2020). Das in diesem Zusammenhang herangezogene „Sequential Exploratory Design“ (Creswell et al. 2007) ist ein MixedMethods-Ansatz, der auf der Analyse von hauptsächlich qualitativen, aber auch quantitativen Daten basiert, die sequentiell gesammelt, priorisiert und in eine oder mehrere Phasen des Forschungsprozesses integriert wurden.

Aus Gründen der Darstellbarkeit werden detaillierte Inhalte zu diesen Ausführungen in diesem Beitrag nicht präsentiert.

\section{Wissensrelevanz in industriellen Rüstprozessen}

Rüstvorgänge erfolgen zwischen dem Ende der Serienproduktion eines Artikels und dem Beginn der Serienproduktion eines weiteren Artikels. Ohne diesen erfolgskritischen Prozess könnte es keine Produktion geben (Kober et al. 2014). Der Komplexitätsgrad industrieller Rüstvorgänge kann je nach Konzeption der Werkzeuge und Maschinen stark variieren. Somit weisen derartige Prozesse, wie sie z.B. an Umform- bzw. Biegemaschinen durchgeführt werden, einen wissensintensiven Charakter auf. 


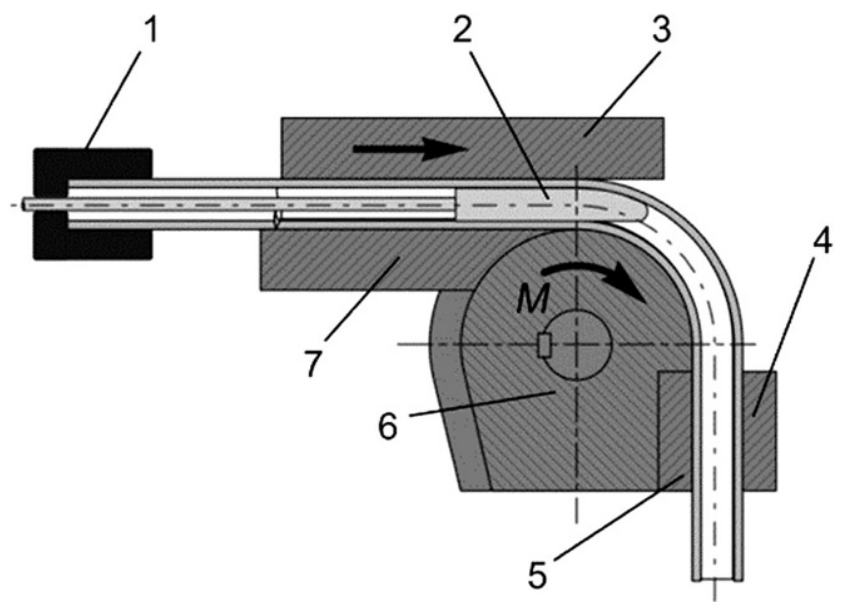

Abb. 1 Werkzeuganordnung des Rotationszugbiegeprozesses: Spannfutter (1), Biegedorn (2), Gegenhalter (3), äußere Spannbacke (4), innere Spannbacke (5), Biegeform (6), Faltenglätter (7) (Quelle: VDI 3430) Fig. 1 Tool arrangement of the rotary draw bending process: collet (1), mandrel (2), pressure die (3), outer clamp die (4), inner clamp die (5), bend die (6), wiper die (7) (source: VDI 3430)

Ein Anwendungsbeispiel stellt das Rotationszugbiegen dar. Nach VDI 3430 (2014) sowie Engel und Mathes (2013) ist das Rotationszugbiegen ein formgebendes Kaltumformverfahren. Die Formgebung des Halbzeugs, das sowohl als Voll- als auch als Hohlprofil gebogen werden kann, wird durch drehende Werkzeugbewegungen und formspeichernde Werkzeuge erreicht. Der Werkzeugaufbau (vgl. Abb. 1) umfasst mit Spannfutter, Biegedorn, Gegenhalter, äußerer und innerer Spannbacke, Biegeform sowie Faltenglätter i.d. R. sieben Werkzeugelemente.

Im Folgenden werden Erkenntnisse über die Praktiken industrieller Rüstvorgänge und die daraus resultierenden Nutzeranforderungen vorgestellt. Diese Erkenntnisse bilden die Grundlage für die Ausarbeitung eines praxisbasierten Modells für betriebliche Rüstprozesse sowie für die Gestaltung einer cyber-physischen Unterstützungssystematik.

\subsection{Charakteristik industrieller Rüstprozesse}

Die Analyse der Praktiken von Maschineneinrichter:innen legen nahe, dass industrielle Rüstvorgänge in sechs voneinander abhängige Phasen unterteilt werden können: Vorbereitung (1), Logistik (2), Rüsten von Werkzeug und Maschine (3), Produktion (4), Inspektion (5) und Programmiterationen (6).

Das Rüsten kann in Abhängigkeit der Komplexität des Rüstvorgangs und der Maschinengröße von einer einzelnen Arbeitsperson oder von zwei oder mehr Mitarbeitern bzw. Mitarbeiterinnen durchgeführt werden. Wissenstransferprozesse treten in dem beschriebenen Einrichtungsszenario insbesondere bei einzeln durchgeführten Arbeitsvorgängen deutlicher hervor als bei kollaborativen Rüstvorgängen.
Um Maschinenstillstandzeiten zu reduzieren, müssen in der Vorbereitungsphase klare strategische Aktivitäten zu den Spezifikationen des Produktionsprozesses sowie zur Einplanung der vorhandenen Ressourcen (Halbfertigteile, Maschinen- und Personalverfügbarkeit) festgelegt werden. Die Schwierigkeit dieses Vorhabens wird durch das folgende Zitat verdeutlicht: „Einerseits muss der Bestand minimiert werden, indem die Produktion variabel gestaltet wird und viele Rüstvorgänge durchgeführt werden. Umgekehrt ist es wichtig, die Gesamtrüstzeiten durch wenige Rüstvorgänge gering zu halten." (T4, Interview).

Während die Vorbereitung ausschließlich als externe bzw. nur bei Maschinenstillstand erfolgende Rüsttätigkeit ausgewiesen wird, kann die Logistik sowohl externe als auch interne, d.h. während der laufenden Maschine durchzuführende Rüstaktivitäten beinhalten. Im Zuge dessen müssen Maschineneinrichter:innen parallel zu sämtlichen Rüstphasen die artikelspezifischen Werkzeuge und Materialien an der zu rüstenden Maschine bereitstellen. Die Analyse der Eye-Tracking-Aufnahmen zeigte, dass logistische Aktivitäten bis zu $21 \%$ der Rüstzeit (gemittelte Rüstzeit von 79 min bei der gleichen Maschine, mit den gleichen Werkzeugen, für das gleiche Produkt, durch Maschinenbediener mit nahezu identischen Erfahrungswerten) einnehmen. Insbesondere können zusätzliche (und vermeidbare) Laufwege zu Störungen einer geordneten Rüstreihenfolge und damit zu Rüstzeiterhöhungen führen.

In der Werkzeug- und Maschineneinrichtphase erfolgt der aus Montage und Demontage unterschiedlicher Komponenten bestehende mechanische Rüstvorgang. Aus der Analyse wurde deutlich, dass mit zunehmender Komplexität der mechanischen Tätigkeiten das „Know how“ der Maschinenbediener für ein erfolgreiches und effizientes Rüsten entscheidend wird. Grundsätzlich qualifiziert das Wissen über den Rüstvorgang die Mitarbeiter, diese Tätigkeiten ohne weitere Einschränkungen durchzuführen. Die Ergebnisse deuten auf den Bedarf einer schrittweisen Anleitung bzw. Rüstinstruktion hin.

Die Phasen der Produktion, Inspektion und Programmiterationen gehen ineinander über. Die Produktionsphase stellt in Bezug auf den tatsächlichen Herstellungsprozess der Artikel keine zeitkritischen und inhaltlichen Herausforderungen dar. Vielmehr nimmt die Inspektion durch Überprüfung der Bauteilqualität in Form einer optischen und taktilen (Lehren-)Messung am gefertigten Erzeugnis eine besondere Rolle ein. Etwaige geometrische Abweichungen nehmen unmittelbar Einfluss auf den Rüstvorgang und die anschließende Maschinenprogrammierung bzw. Programmiteration: „Wenn das Teil nicht lehrenhaltig ist, muss ich in den Prozess eingreifen und Maschinenparameter ändern." (T1, Interview). Nach der Fehlererkennung am Bauteil muss zur Korrektur die entsprechende Zeile im Programmcode der Maschinensteuerung gefunden und in möglichst weni- 
gen Iterationsschritten angepasst werden. Ein zeichnungsgerechtes und fehlerfreies Produkt kann durchaus mithilfe mehrerer bzw. unterschiedlicher Einstellungen zielführend sein.

Für derartig komplexe Vorgänge ist die Darstellung von unterstützenden und simulationsbasierten Informationen sinnvoll. Sofern für komplexe Geometrien und starke Materialabhängigkeiten keine analytischen bzw. mathematischen Lösungen existieren, können bspw. Simulationen mittels Finite-Elemente-Methode (FEM) die Möglichkeit einer näherungsweisen theoretisch und numerisch fundierten Lösung bieten.

\subsection{Abhängigkeit von Wissen}

Die Forschungsergebnisse haben gezeigt, dass industrielle Rüstprozesse in Bezug auf mechanische (z. B. Montage und Demontage der Werkzeuge) und nicht-mechanische (z. B. Interaktion mit der Nutzeroberfläche der Maschine) Arbeitsinhalte sowohl statische als auch dynamische Dimensionen aufweisen. Innerhalb des Rüstprozesses finden sich demnach wiederholende bzw. reproduzierbare (Vorbereitung, Logistik und Inspektion) sowie sehr variable Arbeitsvorgänge (Werkzeug- und Maschinenrüsten) wieder. Während einige Schritte, wie bspw. die Montage des Faltenglätters, aufgrund ihrer einfachen und schnellen Erklärbarkeit - z.B. mithilfe von Checklisten - dokumentiert und damit systematisiert sowie expliziert werden können, sind es wiederum andere nicht. Letzteres liegt insbesondere daran, dass Maschineneinrichter:innen ihre Handlungen aufgrund vermeintlicher Banalität oder einer zu hohen Variabilität oder Komplexität nicht artikulieren können. Schlussendlich wird ersichtlich, dass der Prozess hochgradig vom Wissen der Maschineneinrichter:innen abhängig ist.

Die Literatur weist bereits darauf hin, dass das in diesem Kontext beschriebene Wissen sowohl sehr individuell sein kann als auch sehr schwer zu erfassen und zu teilen ist (Nonaka et al. 2000; Randall et al. 1996). Die Notwendigkeit, einen Wissenstransfer innerhalb der Belegschaft bzw. der Kollegen $\mathrm{zu}$ initiieren, wird u.a. durch das folgende Zitat bekräftigt: „[...] Es fehlt einfach die Dokumentation, sodass das Wissen hauptsächlich, im Kopf der einzelnen Mitarbeiter bleibt. Wenn heute drei Mitarbeiter das Unternehmen verlassen und morgen drei neue eingestellt werden, dann ergibt sich ein massives Problem. " (T4, Interview).

Unerfahrene Mitarbeiter sind davon besonders betroffen. Eine einfache Weitergabe von Wissen stellt durch dessen Vielfältigkeit allerdings eine besondere Herausforderung dar (Ackerman et al. 2013; Randall et al. 1996; Nonaka 1991). Weiterhin kann die persönliche Übermittlung von Wissen einerseits belastend sein („Auf Dauer ist das anstrengend. Ich musste jeden Schritt erklären, was er machen soll.", T14, Interview) und andererseits ein persön- lich empfundenes Alleinstellungsmerkmal gefährden. Unabhängig davon hat sich die gemeinsame Nutzung von Wissen und Expertise als erfolgskritische Grundlage für die Verteilung von Ressourcen herausgestellt. Für eine nutzerzentrierte Konzipierung muss eine Systematik, die für den Wissensaustausch genutzt werden soll, neben einer aktuellen Dokumentation insbesondere verschiedene Informationsformate bereitstellen und dabei dem Prinzip des verkörperten Wissens folgen. Umfassendes Verständnis hinsichtlich der Handhabung bestimmter Arbeitsaufgaben kann erst sichtbar gemacht werden, wenn es in Aktionen beobachtet wird. Die damit einhergehende Komplexität der Wissenserfassung und -weitergabe (Nonaka 1991) verlangt nach innovativen Lösungen. CPS können, ohne dass Wissen in propositionales Wissen übersetzt werden muss, Wege bieten, über die das durch Handlungen verkörperte rüstspezifische Wissen erfasst und dargestellt werden kann (De Carvalho et al. 2018).

\section{Wissenstransfermodell im Rahmen industrieller Rüstprozesse}

Prozessspezifische Modelle, wie das von Shingo (1985) entwickelte Modell des „Single Minute Exchange of Die“ (SMED), teilen den Rüstvorgang zweiteilig in interne und externe Arbeitsinhalte auf. Rüstvorgänge weisen allerdings insbesondere hinsichtlich der angewendeten Praktiken eine weitaus komplexere Struktur auf. Das auf empirischen Daten beruhende Wissenstransfermodell, das in Abb. 2 schematisch dargestellt und dessen wesentliche Elemente nachfolgend beschrieben werden, wurde entwickelt, um den Fortschritt und die Umsetzung neuer und innovativer Technologien zu unterstützen. Es stellt die empirisch erhobenen Daten unter besonderer Berücksichtigung der Wissensträger und -empfänger in einem situativen und organisatorischen Kontext dar. CPS können in Form unterschiedlicher (sensorischer) Endgeräte (z.B. AR-Datenbrillen, 3D-Kameras, Simulationsprogramme etc.) und Schnittstellen (z. B. CAD, OPC UA, Websocket etc.) Informationen automatisch erfassen, verarbeiten und zu konsumierbarem Wissen aufbereiten.

Die Handlungen und Eigenschaften bzw. Fähigkeiten der Wissensakteure sind für die Qualität des Wissenstransfers von wesentlicher Bedeutung. Dabei ist entscheidend, auf welche Art und Weise Wissen dargestellt und dokumentiert sowie in welchem situativen Kontext es transferiert wird. Letzteres umfasst neben der Qualität der dem Prozess zugrunde liegenden Werkzeug- und Materialparameter (A, Abb. 2) insbesondere die Rüstperformance bzw. praxisorientierte Fähigkeiten der Einrichter:innen (B, Abb. 2) sowie deren theoretische Grundlagen in Form von fachspezifischem und analytischem Wissen über den Prozess (C, 


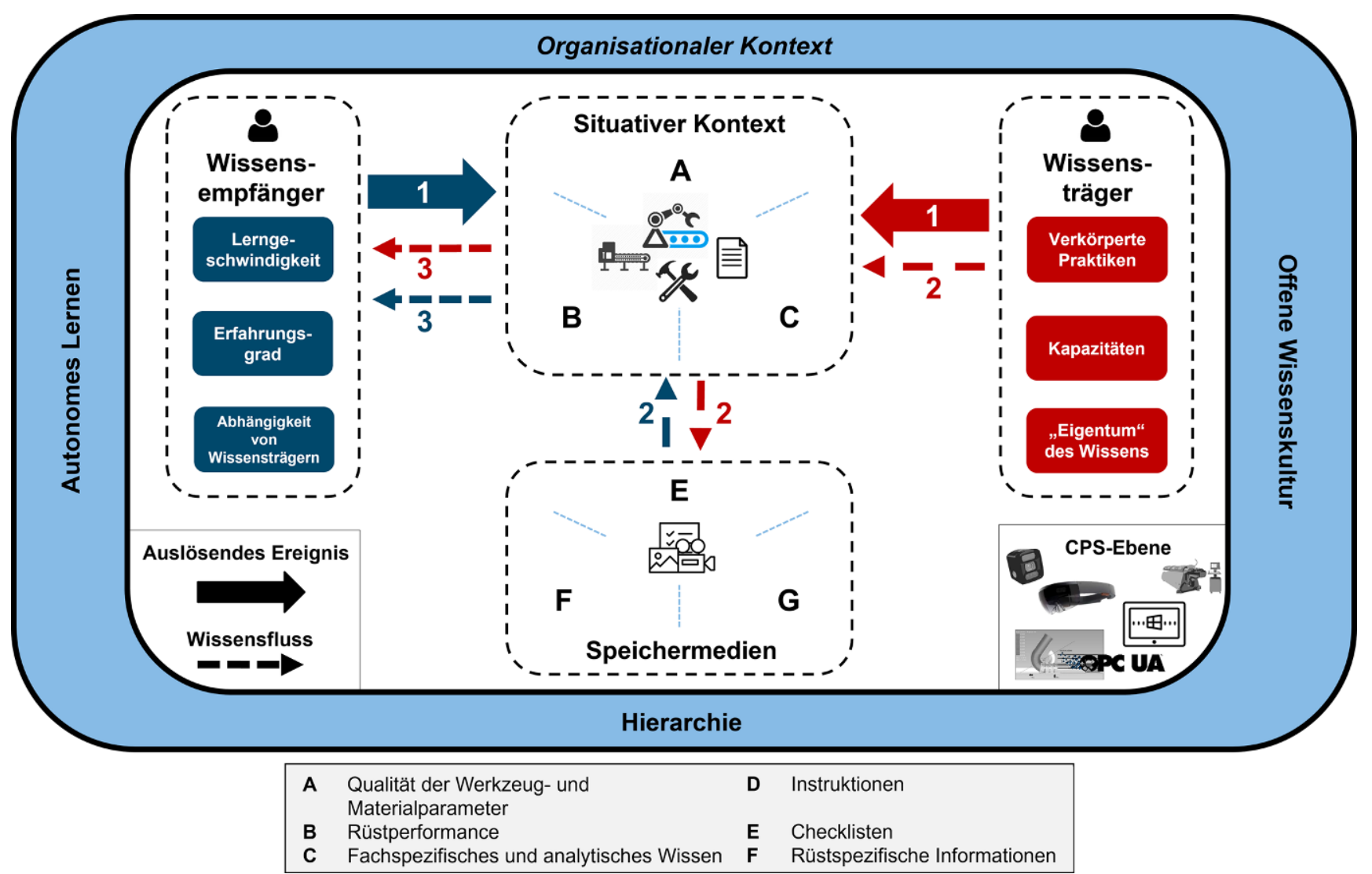

Abb. 2 Wissenstransfermodell im Rahmen industrieller Rüstvorgänge

Fig. 2 Knowledge transfer model in the context of industrial set-up processes

Abb. 2). Dabei sind die Ausführungszeiten für bestimmte (Rüst-)Tätigkeiten, insbesondere manuelle Tätigkeiten, elementar. Diese werden mit den Sollzeiten verglichen, um das Entwicklungs- und Leistungspotenzial zu beurteilen. Gleichzeitig können sie einen Auslöser darstellen, der den Wissensaustausch (Phase 1, Abb. 2) initiiert. Ein wesentlicher Ausgangspunkt eines Wissenstransferansatzes ist die Erkenntnis, dass es zeitliche Unterschiede gibt. Die zeitliche Leistung der Maschineneinrichter:innen kann demnach als Vergleichsbasis herangezogen werden. Wird dieser Vergleich mitsamt der Entwicklung der Rüstzeiten und Fehlerhäufigkeiten eines Bedieners ausgeweitet, können weitere wichtige Rückschlüsse auf die Initiierung eines Wissensaustauschs gezogen werden.

Der organisatorische Rahmen des Modells wird maßgeblich von drei Aspekten gebildet: autonomes Lernen, eine offene Wissenskultur sowie eine gelebte Hierarchie. Erfahrene Mitarbeiter leiten unerfahrene bzw. anzulernende Kollegen zum autonomen Lernen an, nachdem sie ihnen gezeigt haben, wie spezifische Aufgabeninhalte und Verfahrensweisen auszuführen sind. So können die Lernenden Verantwortung für den Lernprozess übernehmen, um sich die Qualifikationen bzw. das Wissen selbst anzueignen. Durch Schulungen und Workshops wird Wissen zudem extern ein- gebracht. Die Analyse hat weiterhin gezeigt, dass Berufsanfänger einen großen Teil ihrer Freizeit dem Erlernen ihres Handwerkszeugs widmen. Gleichzeitig sind die Unternehmen bestrebt, eine offene Wissenskultur zu pflegen, damit die Wissensträger das erworbene Wissen innerhalb des Unternehmens weitergeben und dieses nicht für sich behalten. Dieser Sachverhalt wird durch Teamleiter und Vorgesetzte bekräftigt, wodurch sich ein stark hierarchisch geprägter Charakter abzeichnet. Obwohl beispielsweise neue Arbeitnehmer bei der Gestaltung ihrer Lernprozesse und der Aneignung von gemeinsamem Wissen eine gewisse Autonomie aufweisen, können hierarchische Eingriffe in Form von Vorgaben oder Anweisungen bestimmen, wie Wissen angewendet wird.

Eine weitere organisatorisch geprägte Strategie des Wissenstransfers ist die Förderung der Dokumentation und Lösungsdarstellung von Arbeitsabläufen. Eine entsprechende Dokumentation stellt eine Möglichkeit dar, erfahrungsbasiertes Wissen durch das Teilen von „Know how“ festzuhalten (Ackerman et al. 2013; Randall et al. 1996), um einerseits unerfahrene Einrichter:innen zu unterstützen und andererseits erfahrene Mitarbeiter zu entlasten. Allerdings ist der Dokumentationsprozess zeitintensiv. Außerdem können schriftliche Instruktionen einen kommunikativen und prak- 
tischen Ansatz nicht ersetzen, sondern höchstens unterstützen. CPS können dabei helfen, die Erfassung und Bereitstellung bestimmter Informationsarten zu automatisieren, z. B. durch den Einsatz von AR-Technologien. In der zweiten Phase (2, Abb. 2) des Wissenstransfers ist die Archivierung und Verfügbarmachung von Wissen elementar und erfolgt oftmals durch direkte Kommunikation. Im Schichtbetrieb ist eine direkte Kommunikation allerdings nicht immer oder nur bedingt möglich. Somit kann der Austausch alternativ über Speichermedien asynchron in Form von Schrittfür-Schritt-Instruktionen (D, Abb. 2) oder Checklisten (E, Abb. 2) erfolgen, die durch mediale bzw. bild- und videobasierte Inhalte (F, Abb. 2) ergänzt werden.

Das Wissen, das sich Menschen durch Erfahrungen aneignen, ist implizit mit ihnen selbst verbunden, sodass es in ihren Praktiken verkörpert wird. Diese Art von Wissen lässt sich nur schwer verbal oder mittels Propositionen festhalten bzw. weitergeben. Wissensträger, die Wissen weitergeben sollen, können dafür oftmals nicht genügend Kapazitäten aufbringen. Außerdem wirft bspw. die Aufzeichnung und Weitergabe von erfahrungsbasierten Inhalten Fragen des „Eigentums“ sowie weitere ethische Aspekte auf, die berücksichtigt werden müssen. Daher besteht eine Notwendigkeit darin, die beschriebene Autonomie der Wissenssuchenden zu stärken.

Der Wissenserwerb und die Wissensintegration bilden die dritte Phase (3, Abb. 2) des Wissenstransfers. Wissenssuchende müssen in der Lage sein, neue Inhalte zu lernen, diese mit bekanntem Wissen zu kombinieren und in der Praxis auf Grundlage von gewonnenem Verständnis einzusetzen.

Für Wissensempfänger bzw. -suchende sind insbesondere ihre Lerngeschwindigkeit, ihr Erfahrungsgrad sowie ihre Abhängigkeit vom Wissensträger elementare Aspekte innerhalb des Wissenstransfermodells. Die Ergebnisse deuten darauf hin, dass manche Maschineneinrichter:innen im Vergleich zu anderen z. T. deutlich kürzere Rüstzeiten bei gleicher oder nur geringfügig abweichender Qualität aufweisen. Unabhängig von möglichen Qualifizierungs- bzw. Fortbildungsmaßnahmen ist es nicht vollumfänglich möglich, für sämtliche Mitarbeiter dieselbe Lerngeschwindigkeit zu garantieren. Zu Beginn der Lernphase eines Wissenssuchenden kann dieser je nach Lerngeschwindigkeit sehr stark an die wissenden Personen gebunden sein. Diese Abhängigkeit kann zu Produktionsengpässen führen, wenn die Arbeitsschichten nicht mit den ,richtigen“ Personen besetzt sind. Bei der Interaktion mit einem Wissensträger ist der Grad der Expertise des Wissenssuchenden für den Erfolg des Wissenstransfers entscheidend. Bestimmte prozessrelevante Details werden oftmals gar nicht erwähnt, weil sie für selbstverständlich gehalten werden.

\section{Design eines auf Augmented Reality basierenden Cyber-Physischen Systems für industrielle Rüstprozesse}

Die auf Grundlage der empirischen Daten gewonnenen und durch das Wissenstransfermodell kanalisierten Erkenntnisse führten zur Identifizierung einer Reihe von Anforderungen an ein Unterstützungssystem, das Maschineneinrichter:innen bei industriellen Rüstvorgängen unterstützten soll. Diese Anforderungen wurden durch praxisorientierte Designimplikationen für CPS im beschriebenen Kontext umgesetzt.

\subsection{Designimplikationen}

Insgesamt wurden sechs wesentliche Designimplikationen herausgestellt, die nachfolgend vorgestellt werden. Die Ausführungen zeigen auf, dass die beschriebenen Themenbereiche die von Lee et al. (2015) entwickelte und technisch orientierte 5-Ebenen-Architektur erweitern und konkretisieren können. Die Erkenntnisse beinhalten sozio-technische Designaspekte und Möglichkeiten der Generierung einer wissensorientierten Arbeitsumgebung.

Die Verwendung von Sensoren zur Erfassung von Realdaten (1) bezieht sich im Wesentlichen auf die ,Smart Connected"-Ebene von Lee et al. (2015). Sensorbasierte Bewegungsverfolgung bzw. ,Wegtracking“ kann im Zuge des Rüstvorgangs entstandene Laufwege insbesondere für logistische Aktivitäten aufzeichnen und z. B. in Form eines Spaghetti-Diagramms ausgeben. Weiterhin verhilft eine sensorbasierte Erkennung, bspw. durch eine 3D-Kamera, zur eindeutigen Identifikation der Werkzeuge und zur Bestimmung der Montageposition auf der Maschinenachse. Mithilfe von Sensorik kann außerdem lokales Handlungswissen in Form von Videos aufgezeichnet und weitergegeben werden. Sensorik kann dem Maschineneinrichter bzw. der Maschineneinrichterin also produktspezifische Eigenschaften in Echtzeit visualisieren und damit eine kontextsensitive Interaktion ermöglichen.

Die Nutzung digitaler Simulationen in Situationen, in denen keine realen Daten zur Verfügung stehen (2), kann der Ebene zur Umwandlung von Daten in Informationen (Lee et al. 2015) zugeordnet werden. Durch die Einblendung von 3D-Hologrammen der Maschine bzw. Werkzeuge werden reale (zu rüstende) Elemente durch virtuelle (CAD-)Daten überlagert (AR-Overlay), um dem Nutzer eine eindeutige Funktionsdurchführung zu ermöglichen. Darüber hinaus kann durch virtuelle kinematische Simulationen in der Planungsphase sowie mithilfe der AR-Visualisierungen an der realen Maschine während des Rüstvorgangs eine kollisionsfreie Montage der Werkzeuge gewährleistet werden. Die virtuelle Generierung von Prozessdaten kann 
also die Übersetzung bestimmter Aspekte des lokalen Wissens in propositionale Inhalte ermöglichen.

Die „Cyber“-Ebene von Lee et al. (2015) beinhaltet die Verknüpfung digitaler Daten zu Arbeitspraktiken bzw. erfahrungsbasiertem Wissen (3). Dabei geht es im Wesentlichen darum, einen digitalen Zwilling bzw. ein Zwillingsmodell als virtuelle Repräsentation der realen Arbeitsabläufe für den Umgang mit Werkzeug- und Maschinenkomponenten zu erzeugen. Virtuelle Daten werden in einer AR-Animation innerhalb des Unterstützungssystems erstellt und in einem Dashboard visualisiert. Dabei ist eine lernfreundliche Umgebung durch eine realitätsnahe und kontextsensitive Datenaggregation sowie einen hohen und problemorientierten Detailgrad mit einem möglichst geringen Komplexitätsgrad herzustellen.

Die Speicherung von Wissen sowie die individuelle und unabhängige Zugänglichkeit von multimedialen Inhalten stellt innerhalb einer industriellen Umgebung einen entscheidenden Vorteil dar (Caudell und Mizell 1992). Fortschrittliche (technologische) Systeme weisen ein großes Potenzial zu einer lückenlosen, detailgetreuen und aktuellen Informationsdarstellung auf (Paelke und Röcker 2015). Die Visualisierung von Rüstschritten wird auf der zweiten Ebene der CPS-Architektur initiiert und auf der vierten Ebene, der Kognitionsebene, mittels einer AR-Anwendung zu einer vollständigen Rüstinstruktion zusammengefasst. Auf Basis dieser interaktiven Anweisung stehen Rüstinformationen unabhängig von einer personellen Verfügbarkeit zur Verfügung. Somit wird auf Basis der erfassten und visualisierten Rüstinformationen ein Wissenstransfer unterstützt (4).

Interaktive Checklisten verhelfen zugunsten einer Fehlervermeidung zur Verifizierung oder Falsifizierung der physischen Verfügbarkeit oder der Soll-Montageposition von Werkzeugen sowie zur schrittweisen Darstellung der Arbeitsaufgabe in Form von textlichen sowie bild- und videobasierten Inhalten (Fillatreau et al. 2013). Die beschriebenen Erkenntnisse stimmen mit der Konfigurationsebene von Lee et al. (2015) überein. Diese Unterstützung der Datenkonfiguration (5) kann als iterativer und lernförderlicher Ansatz verstanden werden, der durch rollierende Anpassungsmöglichkeiten der Arbeitsabläufe einen BestPractice-Ansatz verfolgt.

Eine Implikation, die nicht explizit von Lee et al. (2015) benannt wird, dafür aber sämtliche Ebenen durchdringt, betrifft die Interaktionsherausforderungen, denen sich ein Einrichter bzw. eine Einrichterin im Produktionsalltag stellen muss. Unter Berücksichtigung dieser Herausforderungen, die sich hauptsächlich aus den Interaktionen zwischen Nutzern und dem System in der Umgebung ergeben, in der das System eingesetzt wird, soll eine praktikable Interaktion im industriellen Kontext (6) gewährleistet sein. Das Unterstützungssystem sollte relevante Informationen zunächst zeitnah und mit minimalen Fehlern bereitstellen, um einem Wissensdefizit sowie einer fehlerintoleranten Produktionsumgebung entgegenzuwirken. Eine angemessene Visualisierung der Inhalte bedarf sowohl einer schrittweisen Erfassung als auch Wiedergabe eines multimedialen Datenmaterials aus der Ich-Perspektive. Diese Anforderungen können bspw. mithilfe von gestengesteuerten Head-Mounted Displays realisiert werden. HMDs bzw. Datenbrillen gewährleisten, dass der Nutzer beide Hände einsetzen kann, um Rüstoperationen auszuführen. Weiterhin sind diese mobilen Systeme in begrenzten Arbeitsräumen von Vorteil. Einerseits muss das System robust genug sein, um etwaige Unfälle ohne funktionelle Ausfälle zu überstehen. Andererseits dürfen Umgebungsbedingungen, wie industriebedingter Lärm, die Arbeitssicherheit sowie die Funktion bzw. Interaktion mit dem Gerät nicht beeinträchtigen. In diesem Zusammenhang verhilft geeignete Sensorik zugunsten einer effizienten, fehler- und beanspruchungsminimalen Instruktion dazu, den Arbeitsbereich zu überwachen und etwaige Unsicherheiten seitens des Nutzers zu verringern. Eine schrittweise Erfassung der erforderlichen Arbeitsschritte kann zudem in Kombination mit einer Ortungsfunktion als Grundlage für Optimierungsmaßnahmen des gesamten Rüstprozesses dienen.

\subsection{Technische Umsetzung der Designimplikationen}

Das Potenzial neuer Technologien zur Verbesserung von Wissenstransferprozessen, wie z.B. durch AR, wurde bereits von De Carvalho et al. (2018) ausführlich diskutiert. Neben einer geeigneten Auswahl der Unterstützungssysteme und deren Einbindung in die technische Infrastruktur bedarf es einer reibungslosen Integration der Nutzerinteraktionen mit der Technologie während des gesamten Rüstvorgangs. Um den beschriebenen Interaktionsanforderungen und Designimplikationen bestmöglich gerecht zu werden, wurde eine prototypische Rüst-Applikation bzw. -Software für die AR-Datenbrille ,Microsoft HoloLens“ der ersten Generation umgesetzt (vgl. I, Abb. 3). Die Microsoft HoloLens ist eine binokulare Windows-10-betriebene Mixed-Reality-Brille. Durch eine eigene (holografische) Prozessoreinheit (Holographic Processing Unit, HPU) kann die HoloLens autark verwendet werden. Nutzer können durch Natural-User-Interfaces mit hochauflösenden 3DHologrammen, die in die direkte bzw. reale Umgebung des Anwenders projiziert werden, über Sprach- und Gestensteuerung interagieren (N.N. 2019). Visualisierungen werden in den aktuellen Kontext eingebettet, indem sie mit der Umgebung verschmelzen und kontextabhängige Anweisungen und Informationen anzeigen, sodass die Anweisungen schnell gefunden und umgesetzt werden können. Ein weiterer Vorteil des Einsatzes der HoloLens ist 

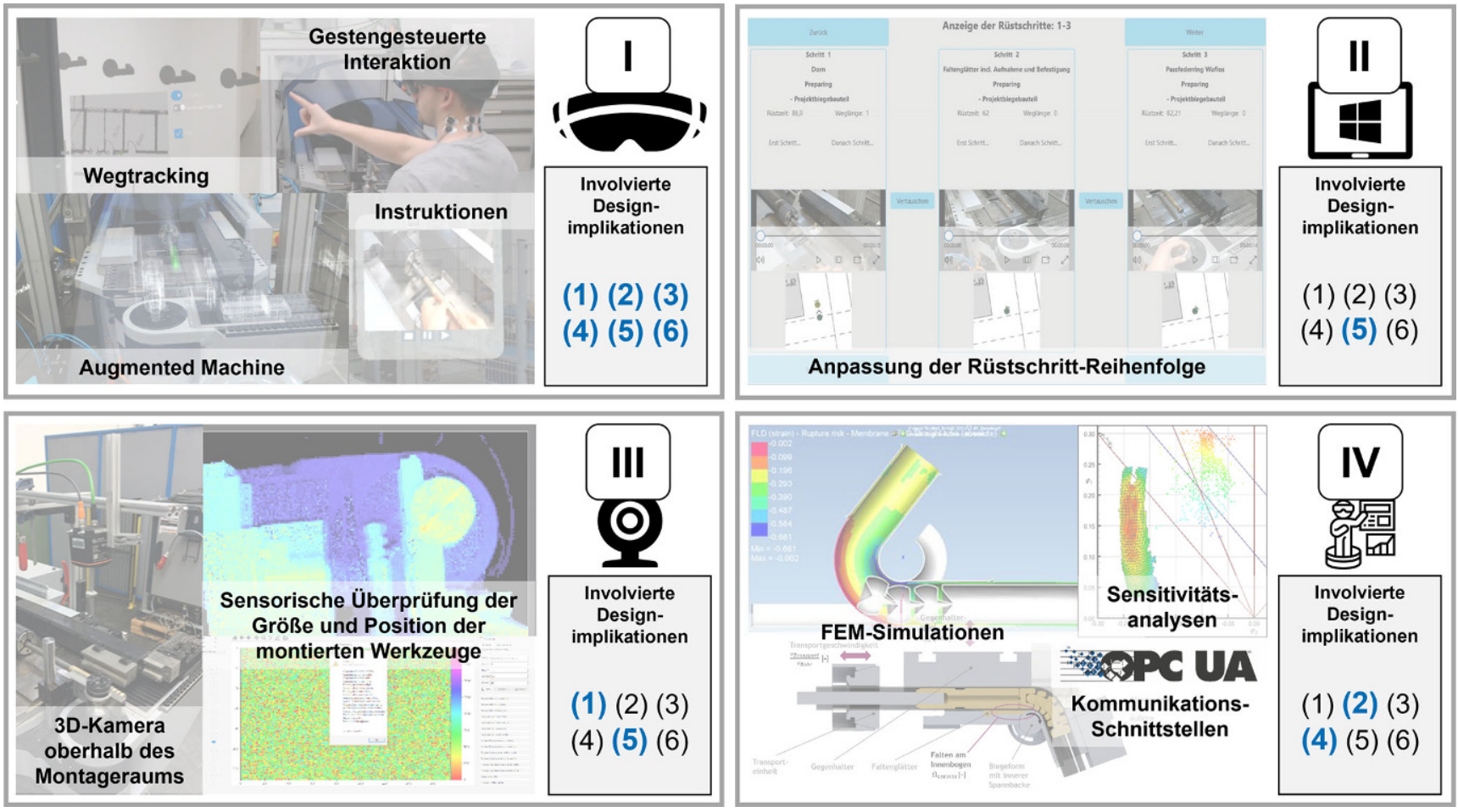

Abb. 3 Exemplarischer Überblick über die abgeleiteten Gestaltungsimplikationen in Bezug auf deren Umsetzung in CPS

Fig. 3 Exemplary overview of derived design implications with regard to their implementation in CPS

der spielerische Umgang und die Interaktion mit dem Gerät. Somit wird einem sogenannten „Gamification“-Effekt gefolgt, der positiv zum Wissenstransfer beiträgt (Vasilescu et al. 2014). Feedback-Mechanismen, wie die Erfassung und Rückmeldung einer 3D-Kamera zu Montagepositionen und Werkzeugabmessungen (vgl. III, Abb. 3) oder die Aufzeichnungsmöglichkeit von zurückgelegten Wegen durch die HoloLens (vgl. I, Abb. 3), tragen außerdem zu einem effektiven Lernprozess bei. Auf Letzteres greift ein Tablet-Client bzw. ein sogenannter „Editor“ zu, der dazu beiträgt, dass die aggregierten Rüstdaten durch Änderung der Rüstschritt-Reihenfolge angepasst werden können (vgl. II, Abb. 3). Um darüber hinaus auch theoretische und physikalische Rahmenbedingungen des Rüstprozesses einzubinden, können FEM-Simulationen wichtige Informationen über Materialverhalten liefern. Durch den Zugriff auf die Maschinensteuerung und die Anzeige der Maschinenparameter, die über eine OPC-UA-Schnittstelle kommunizieren, können Einstellanweisungen abgeleitet und auf nachfolgende Rüstprozesse übertragen werden (vgl. IV, Abb. 3).

\section{Diskussion und Ausblick}

Die Ergebnisse zeigen, dass industrielle Rüstvorgänge sehr wissensintensive und komplexe Prozesse darstellen. Nur zwischen implizitem und explizitem Wissen bzw. „Know how“ und „Know why“ zu unterscheiden, reicht nicht aus (Schmidt 2012). Wissen existiert innerhalb der Situationen, in denen man es zu explizieren versucht (Sacks 1995). Die Detailgenauigkeit und die Bereitschaft Wissen zu teilen, variiert in Abhängigkeit der beteiligten Akteure. Daher sollte Wissen in prozessrelevante Elemente spezifiziert und mit begleitenden technologischen Maßnahmen zum Wissenstransfer gestützt werden. Zwar ähneln die Erkenntnisse hinsichtlich des Transfers von Wissen denen aus Studien anderer Fachgebiete, wie z. B. über Diagnose- oder Pflegetätigkeiten in medizinischen Arbeitsumgebungen (Bardram und Bossen 2005). Allerdings grenzen sich die hier dargestellten Ergebnisse davon ab: Das Wissen konzentriert sich auf wenige Personen innerhalb einer Organisation. Wenn das Wissen dieser Personen nicht greifbar ist, z. B. durch Krankheit oder Ausscheiden aus dem Unternehmen, können für das Unternehmen prozessbezogene und ökonomische Defizite entstehen.

Designimplikationen für ein AR-basiertes CPS, das im beschriebenen Rüstkontext eingesetzt und zu einem effektiveren Wissenstransfer seitens der Maschineneinrichter:innen beitragen soll, wurden bisher insbesondere hinsichtlich verkörperter Handlungen noch nicht umfassend erforscht (De Carvalho et al. 2018). Der vorliegende Forschungsansatz zielt darauf ab, den diesbezüglichen Stand der Technik voranzutreiben. Design und Sensorik unter- 
stützen Bediener dabei, verschiedene Rüstszenarien unterschiedlicher Präsentationsformate (Texte, Bilder, Videos und AR-Overlays) kontextspezifisch zu erfassen, zu verarbeiten und $\mathrm{zu}$ visualisieren, um darauf aufbauend Lern- und Verbesserungsprozesse anzustoßen. Mechanische Arbeitsschritte weisen zumeist prozessuale Wissensbestandteile auf, die durch verkörperte Handlungen repräsentiert werden. Nicht-mechanische Rüstvorgänge können durch das Vorhandensein bzw. die Darstellung von propositionalem Wissen beschrieben werden. Wissen, das vermeintlich irrelevant, selbstverständlich und banal erscheint, jedoch oftmals elementare Erfahrungswerte beinhaltet, kann durch die CPS-Systematik auf Grundlage der sensorischen Informationen mitsamt einer kontextualisierten Visualisierung verfügbar gemacht werden. Der Einsatz von AR erlaubt es dem Nutzer in diesem Zusammenhang, digitale und reale bzw. physische Inhalte zu kombinieren. Die Infrastruktur der Daten und Speichermedien muss dabei jeweils an spezifische Situationen und den organisatorischen Rahmen anpassbar sein, damit relevante Instruktionen in den situativen Kontext eingebettet werden können. Somit können durch die Verbesserung der Arbeitsgenauigkeit und Aktualität der Informationen Trial-and-Error-Phasen verkürzt und reduzierte Rüstzeiten generiert werden. Zu erwähnen ist an dieser Stelle außerdem, dass der Trend zur Dokumentation jedoch nicht dazu führt, dass Arbeitnehmer entbehrlich werden. Die Forschungsarbeit hat gezeigt, dass Organisationen erfahrene Arbeitskräfte vielmehr wertschätzen und versuchen, sie an das Unternehmen zu binden.

Ein für die Entwicklung des Wissenstransfermodells entscheidender Schritt war die Identifizierung relevanter Akteure und die Erläuterung, wie sich die gemeinsame Nutzung von Wissen auf ihre Aktivitäten innerhalb des Rüstprozesses unter Berücksichtigung sozialer, organisatorischer und situativer Aspekte bezieht. Der Mensch stellt den Dreh- und Angelpunkt des Wissenstransfers dar. Wie in Abb. 2 dargestellt, findet ein Austausch von Wissen zwischen mindestens zwei Akteuren statt: einer wissenden Person und der Person, die von ihr lernt (Nonaka und Von Krogh 2009). Entweder kann ein Wissenssuchender eine wissende Person aufgrund seines bestehenden Wissensbedarfs bewusst aufsuchen (Pull-Prinzip) oder eine wissende Person erkennt einen potenziellen Bedarf des Wissenssuchenden und sieht sich im Stande, diesen mit Wissen zu versorgen (Push-Prinzip).

Die in diesem Zusammenhang stattfindenden Lernprozesse werden in erster Linie durch direktes, unmittelbares und persönliches Feedback gesteuert. Mit Hilfe des Feedbacks ist es möglich, die jeweilige Tätigkeit zu reflektieren. Nach Banker et al. (1993) führt dieser Sachverhalt zu „Know why“, der wiederum eine Phase im Lernprozess anstößt, in der autonomes Lernen das persönliche Feedback weitgehend ersetzen kann. An einem bestimmten Punkt wurde genug verstanden, um ein abstraktes Verständnis der Vorgänge aufzuweisen, das auf eine Vielzahl von Situationen im Produktionsprozess angewendet werden kann. CPS setzen daran an, indem das Feedback aus dem System selbst konsumiert wird und ortsunabhängig ist. Ein weiterer wichtiger Aspekt ist die Bedeutsamkeit des Verständnisses physikalischer Prinzipien, die den grundlegenden Mechanismen des Rüstprozesses zugrunde liegen. Dieser Aspekt deckt sich mit der Auffassung der CLT zur Aneignung der „Germane load“, um das Wissen auf andere, ähnliche Prozesse zugunsten einer kognitiven Belastungsreduktion zu übertragen.

Mithilfe der CPS-Technologie bzw. des Zusammenspiels von kontextualisierten Visualisierungen, sensorischer Unterstützung und der kombinierten Nutzung von virtuellen und realen Prozessdaten können die im Rahmen eines Rüstvorgangs beobachteten Mechanismen des Wissenstransfers auf eine neue Art und Weise unterstützt und weiterentwickelt werden. Demgegenüber weisen CPS allerdings auch Einschränkungen auf. Neben Ungenauigkeiten bei der sensorischen Umgebungserfassung und den daraus resultierenden Abweichungen bei der Darstellung von Rüstinstruktionen, können weiterhin Unsicherheiten bezüglich des Datenschutzes entstehen. Die sensorische Erfassung von Rüstvorgängen durch die HoloLens und die 3D-Kamera sowie die Möglichkeiten zur Auswertung von Rüstdaten mit Hilfe des Tablet-Clients bzw. Editors steigern die Transparenz von Rüstdaten und lassen Rückschlüsse auf die Performance des Einrichters und der Einrichterin zu.

Die Ergebnisse liefern in jedem Fall eine detaillierte Darstellung der Praktiken der Maschineneinrichter für Rüstvorgänge an Biege- bzw. Umformmaschinen, die in einem CPS zur Rüstunterstützung von Biegemaschinen umgesetzt wurden. Der Ansatz und die Konzeption orientieren sich zwar an einem spezifischen Anwendungsfall, allerdings können die Erkenntnisse auch auf ähnliche Kontexte, wie z.B. das Rüsten von Stanzmaschinen, übertragen und darin eingesetzt werden. Die Forschung zum Einsatz AR- und sensorbasierter CPS sollte zukünftig auf weitere Wissensgebiete übertragen werden, um die Potenziale und Grenzen derartiger Technologien im Rahmen von Wissenstransferprozessen besser zu verstehen. Außerdem sollten, basierend auf bisherigen Erkenntnissen (Abele und Kluth 2021; Paelke und Röcker 2015; Monostori 2014), weitere Potenziale und Herausforderungen auch hinsichtlich der Nutzerakzeptanz und der ergonomischen Limitierungen zum Umgang mit neuartiger Software und Hardware (z.B. AR-Brillen) aufgedeckt werden.

Funding Open Access funding enabled and organized by Projekt DEAL.

Open Access Dieser Artikel wird unter der Creative Commons Namensnennung 4.0 International Lizenz veröffentlicht, welche die Nut- 
zung, Vervielfältigung, Bearbeitung, Verbreitung und Wiedergabe in jeglichem Medium und Format erlaubt, sofern Sie den/die ursprünglichen Autor(en) und die Quelle ordnungsgemäß nennen, einen Link zur Creative Commons Lizenz beifügen und angeben, ob Änderungen vorgenommen wurden.

Die in diesem Artikel enthaltenen Bilder und sonstiges Drittmaterial unterliegen ebenfalls der genannten Creative Commons Lizenz, sofern sich aus der Abbildungslegende nichts anderes ergibt. Sofern das betreffende Material nicht unter der genannten Creative Commons Lizenz steht und die betreffende Handlung nicht nach gesetzlichen Vorschriften erlaubt ist, ist für die oben aufgeführten Weiterverwendungen des Materials die Einwilligung des jeweiligen Rechteinhabers einzuholen.

Weitere Details zur Lizenz entnehmen Sie bitte der Lizenzinformation auf http://creativecommons.org/licenses/by/4.0/deed.de.

\section{Literatur}

Abele ND, Kluth K (2020) Beanspruchungsbezogene Evaluierung ARbasierter versus papierunterstützter Rüstinstruktionen zur Einrichtung von Industriemaschinen. In: Proceedings des 66. Frühjahrskongresses der Gesellschaft für Arbeitswissenschaft. GfA-Press, Dortmund

Abele ND, Kluth K (2021) Strain-related evaluation of an AR-based cyber-physical production system for setting up industrial machines. In: Black N, Neumann P, Noy I (Hrsg) Sector based ergonomics. Proceedings of the 21 st congress of the international ergonomics association (IEA 2021), Bd. III. Springer, Cham, S $355-362$

Abele ND, Hoffmann S, Kuhnhen C, Ludwig T, Schäfer W, Schweitzer M, Wulf V (2016) Supporting the set-up processes by cyber elements based on the example of tube bending. In: Mayr $\mathrm{CH}$, Pinzger M (Hrsg) INFORMATIK 2016. GI-edition lecture notes in Informatics proceedings. Gesellschaft für Informatik, Bonn, S $1627-1636$

Ackerman MS, Halverson C (2004) Sharing expertise: the next step for knowledge management. In: Huysman M, Wulf V (Hrsg) Social capital and information technology. MIT Press, London, S 273-299

Ackerman MS, Dachtera J, Pipek V, Wulf V (2013) Sharing knowledge and expertise: the CSCW view of knowledge management. Comput Support Coop Work 22:531-573

Anderson-Levitt KM (2006) Ethnography. In: Green JL, Camilli G, Elmore PB, Skukaukaiti A, Grace E (Hrsg) Handbook of complementary methods in education research. Lawrence Erlbaum, Mahwah, S 279-298

Argote L, Ingram P, Levine JM, Moreland RL (2000) Knowledge transfer in organizations: learning from the experience of others. Organ Behav Hum Decis Process 82(1):1-8

Banker RD, Potter G, Schroeder RG (1993) Reporting manufacturing performance measures to workers: an empirical study. J Manag Account Res 5(1):33-55

Bardram J, Bossen C (2005) Mobility work: the spatial dimension of collaboration at a hospital. Comput Support Coop Work $14: 131-160$

Benford S, Mariani JA, Moldes LN, Prinz W, Rodden T (1993) MOCCA: an environment for CSCW applications. In: Proceedings of the conference on organizational computing systems (COCS). ACM, New York, S 172-177

Brown JS, Duguid P (1994) Borderline issues: social and material aspects of design. Human-computer Interact 9(1):3-36

Caudell TP, Mizell DW (1992) Augmented reality: an application of heads-up display technology to manual manufacturing processes. In: Proceedings of the twenty-fifth hawaii international conference on system sciences (HICSS). IEEE, New York, S 659-669
Clarke K, Hughes J, Martin D, Rouncefield M, Sommerville I, Gurr C, Hartswood M, Procter R, Slack R, Voss A (2003) Dependable red hot action. In: Kuutti K, Karsten EH, Fitzpatrick G, Dourish P, Schmidt K (Hrsg) Proceedings of the eighth European conference on computer-supported cooperative work (CSCW). Springer, Dordrecht, S 61-80

Creswell JW, Clark VLP, Gutmann M, Hanson WE (2007) An expanded typology for classifying mixed methods research into designs editors' introduction. In: Handbook of mixed methods in social and behavioral research. SAGE, Thousand Oaks, S 209-240

De Carvalho AFP, Hoffmann S, Abele ND, Schweitzer M, Tolmie P, Randall D, Wulf V (2018) Of embodied action and sensors. Knowledge and expertise sharing in industrial set-up. Comput Support Coop Work 27(3-6):875-916

De Michelis G, Grasso MA (1994) Situating conversations within the language/action perspective: the Milan conversation model. In: Proceedings of the 1994 ACM conference on computer-supported cooperative work (CSCW). ACM, New York, S 89-100

Diggins T, Tolmie P (2003) The 'adequate' design of ethnographic outputs for practice: some explorations of the characteristics of design resources. Pers Ubiquitous Comput 7(3-4):147-158

Divitini M, Salè GO, Pozzoli A, Simone C (1993) Supporting the dynamics of knowledge sharing within organizations. In: Proceedings of the conference on organizational computing systems (COCS). ACM, New York, S 178-183

Dörner R, Broll W, Grimm P, Jung B (2019) Virtual und Augmented Reality (VR/AR). Springer, Berlin, Heidelberg

Engel B, Mathes C (2013) Erweiterung der Prozessfähigkeit des Rotationszugbiegens durch ein alternatives Faltenglätterkonzept. Europäische Forschungsgesellschaft für Blechverarbeitung e.V. (EFB), Hannover

Fillatreau P, Fourquet JY, Le Bolloc'h R, Cailhol S, Datas A, Puel B (2013) Using virtual reality and 3D industrial numerical models for Immersive interactive checklists. Comput Ind 64(9): 1253-1262

Fraga-Lamas P, Fernández-Caraméz TM, Blanco-Novoa Ó, VilarMontesinos MA (2018) A review on industrial augmented systems for the industry 4.0 shipyard. IEEE Access 6(2018): 13358-13375

Frigo MA, da Silva ECC, Barbosa GF (2016) Augmented reality in aerospace manufacturing: a review. J Ind Intell Inf 4(2):125-130

Gallagher S (2010) Merleau-Ponty's phenomenology of perception. Topoi Int Rev Philos 29(2):183-185

Goodwin C (1994) Professional vision. Am Anthropol 96(3):603-633

Heath C, Luff P (1991) Disembodied conduct: communication through video in a multi-media office environment. In: Proceedings of the SIGCHI conference on human factors in computing systems. ACM, New York, S 99-103

Hegenberg J, Schmidt L (2021) Augmented-Reality-basierte Assistenz für das Anlernen Manueller und Roboterunterstützter Montageprozesse. Z Arb Wiss. https://doi.org/10.1007/s41449-02100278-3

Hermanowicz JC (2002) The great interview: 25 strategies for studying people in bed. Qual Sociol 25(4):479-499

Hickley A (2016) Verbatim, intelligent verbatim or edited transcription? http://penguin-transcription.co.uk/transcription-typeverbatim-intelligent-verbatim-or-edited/. Zugegriffen: 20. Okt. 2018

Hoffmann S, De Carvalho AFP, Abele ND, Schweitzer M, Tolmie P, Wulf V (2019) Cyber-physical systems for knowledge and expertise sharing in manufacturing contexts: towards a model enabling design. Comput Support Coop Work 28:469-509

Holtzblatt K, Jones S (2017) Contextual inquiry: a participatory technique for system design. In: Schuler D, Namioka A (Hrsg) Participatory design: principles and practices. Lawrence Erlbaum, Hillsdale, S 177-210

Hořejší P (2015) Augmented reality system for virtual training of parts assembly. 25th DAAAM international symposium on intelligent manufacturing and automation. Procedia Eng 100(2015):699-706 
Kendon A (1990) Conducting interaction: patterns of behavior in focused encounter. Cambridge University Press, Cambridge

Klopfer E, Perry J, Squire K, Jan M (2005) Collaborative learning through augmented reality role playing. In Proceedings of the 2005 Conference on Computer Support for Collaborative Learning (CSCL): Learning 2005: The Next 10 Years! International Society of the Learning Sciences, Taipei, S 311-315

Kober M, Scheve J, Dahm A (2014) Rüstoptimierung - Das Ziel sind kurze Durchlaufzeiten. Betriebsprax Arbeitsforsch 222:10-15

Lee S, Akin Ö (2011) Augmented reality-based computational fieldwork support for equipment operations and maintenance. Autom Constr 20(4):338-352

Lee J, Bagheri B, Kao HA (2015) A cyber-physical systems architecture for industry 4.0-based manufacturing systems. Manuf Lett 3:18-23

Loch F, Quint F, Brishtel I (2016) Comparing video and augmented reality assistance in manual assembly. In: Proceedings of the 12th international conference on intelligent environments. IEEE, New York, S 147-150

Ludwig T, Kotthaus C, Stein M, Durt H, Kurz C, Wenz J, Doublet T, Becker M, Pipek V, Wulf V (2016) Arbeiten im Mittelstand 4.0 KMU im Spannungsfeld des digitalen Wandels. HMD 53(1):1-16

Lynch M (1997) Scientific practice and ordinary action: Ethnomethodology and social studies of science. Cambridge University Press, Cambridge

Mainwaring SD, Lee CP (2017) Turnover and the model of coordinated action (MoCA). In: Companion of the 2017 ACM conference on computer-supported cooperative work and social computing (CSCW). ACM, New York, S 239-242

Mengis J, Eppler MJ (2012) Visualizing instead of overloading: exploring the promise and problems of visual communication to reduce information overload. In: Strother JB, Ulijn JM, Fazal Z (Hrsg) Information overload. John Wiley \& Sons, Hoboken, S 203-229

Monostori L (2014) Cyber-physical production systems: roots, expectations and R\&D challenges. Procedia CIRP 17:9-13

Muller MJ, Kuhn S (1993) Participatory design. Commun ACM 36(6):24-28

N.N. (2019) HoloLens-Hardware (1. Generation). https://docs. microsoft.com/de-de/hololens/hololens1-hardware. Zugegriffen: 16. Sept. 2019

Nielsen J, Molich R (1990) Heuristic evaluation of user interfaces. In: Proceedings of the SIGCHI conference on human factors in computing systems. ACM, New York, S 249-256

Nonaka I (1991) The knowledge-creating company. Harvard Business Review Classics, Brighton

Nonaka I (1994) A dynamic theory of organizational knowledge creation. Organ Sci 5(1):14-37

Nonaka I, Takeuchi H (1995) The knowledge-creating company: how Japanese companies create the dynamics of innovation. Oxford University Press, New York, Oxford

Nonaka I, Von Krogh G (2009) Tacit knowledge and knowledge conversion: controversy and advancement in organizational knowledge creation theory. Organ Sci 20(3):635-652

Nonaka I, Toyama R, Konno N (2000) SECI, Ba and leadership: a unified model of dynamic knowledge creation. Long Range Plann 33(1):5-34

Ong SK, Yuan ML, Nee AYC (2008) Augmented reality applications in manufacturing: a survey. Int J Prod Res 46(10):2707-2742

Paelke V, Röcker C (2015) User interfaces for cyber-physical systems: challenges and possible approaches. Springer, Cham

Park M, Schmidt L, Schlick C, Luczak H (2007) Design and evaluation of an augmented reality welding helmet. Hum Fact Ergonom Manufact 17(4):317-330
Patterson RE, Blaha LM, Grinstein GG, Liggett KK, Kaveney DE, Sheldon KC, Havig PR, Moore JA (2014) A human cognition framework for information visualization. Comput Graph 42:42-58

Peinl R (2006) A knowledge sharing model illustrated with the software development industry. In: Lehner F, Nösekabel H, Kleinschmidt P (Hrsg) Multikonferenz Wirtschaftsinformatik 2006. GITO, Berlin, S 389-401

Polanyi M (1967) Sense-giving and sense-reading. Philosophy 42(162): 301-325

Pollalis C, Fahnbulleh W, Tynes J, Shaer O (2017) Holomuse: enhancing engagement with archaeological artifacts through gesturebased interaction with holograms. In: Proceedings of the tenth international conference on tangible, embedded, and embodied interaction (TEI). ACM, New York, S 565-570

Raelin JA (1997) A model of work-based learning. Organ Sci 8(6): 563-578

Randall D, O'Brien J, Rouncefield M, Hughes JA (1996) Organisational memory and CSCW: supporting the 'Mavis phenomenon'. In: Proceedings of the sixth Australian conference on computerhuman interaction. IEEE, New York, S 26-33

Ryle G (1945) Knowing how and knowing that: the presidential address. Proc Aristot Soc 46(1945-1946):1-16

Ryle G (2009) The concept of mind. Routledge, Oxon, Abingdon

Sacks H (1995) Lectures on conversation. Blackwell Publishers, Cambridge

Salvador T, Mateas M (1997) Introduction to design ethnography. In: Extended abstracts on human factors in computing systems. ACM, New York, S 166-167

Schmidt K (2012) The trouble with "tacit knowledge". Comput Support Coop Work 21(2-3):163-225

Schmidt K (2014) The concept of 'practice': what's the point? In: Rossitto C, Ciolfi L, Martin D, Conein B (Hrsg) Proceedings of the 11th international conference on the design of cooperative systems. Springer, Cham, S 427-444

Shingo S (1985) A revolution in manufacturing: the SMED system. CRC Press, Boca Raton

Sweller J (1994) Cognitive load theory, learning difficulty, and instructional design. Learn Instr 4(4):295-312

Tang A, Neustaedter C, Greenberg S (2007) Videoarms: embodiments for mixed presence groupware. In: Bryan-Kinns N, Blanford A, Curzon P, Nigay L (Hrsg) People and computers XX—engage. Springer, London, S 85-102

Tergan SO, Keller T (2005) Knowledge and information visualization: searching synergies. Springer, Berlin, Heidelberg

Vasilescu B, Serebrenik A, Devanbu P, Filkov V (2014) How social Q\&A sites are changing knowledge sharing in open source software communities. In: Proceedings of the 17th ACM conference on computer-supported cooperative work \& social computing (CSCW). ACM, New York, S 342-354

VDI 3430 (2014) Rotationszugbiegen von Profilen. Beuth, Berlin, Köln, Frankfurt a.M

Wittgenstein L (1953) Philosophical investigations. Blackwell, London

Wulf V, Müller C, Pipek V, Randall D, Rohde M, Stevens G (2015) Practice-based computing: empirically grounded conceptualizations derived from design case studies. In: Wulf V, Schmidt K, Randall D (Hrsg) Designing socially embedded technologies in the real-world. Springer, London, S 111-150

Zidek K, Pitel J, Adamek M, Lazorik P, Hosovsky A (2020) Digital twin of experimental smart manufacturing assembly system for industry 4.0 concept. Sustainability 12(9):3658 